\title{
Декслансопразол MB
}

\section{В ЛЕЧЕНИИ ГАСТРОЭЗОФАГЕАЛЬНОЙ РЕФЛЮКСНОЙ БОЛЕЗНИ}

\section{М.В. ЛЕОНОВА, Э.Э. АЛИМОВА}

Федеральное государственное бюджетное образовательное учреждение высшего образования «Российский национальный исследовательский медицинский университет имени Н.И. Пирогова» Министерства здравоохранения Российской Федерации: 117997, г. Москва, ул. Островитянова, д. 1

\section{Информация об авторах:}

Леонова Марина Васильевна - д.М.н., профессор, чл.-корр. РАЕН, клинический фармаколог, член Межрегиональной общественной организации «Ассоциация клинических фармакологов России»; тел.: +7 (915) 320-43-79; e-mail: anti23@mail.ru

Алимова Эльмира Эрфановна - К.м.н., доцент кафедры клинической фармакологии лечебного факультета Федерального государственного бюджетного образовательного учреждения высшего образования «Российский национальный исследовательский медицинский университет имени Н.И. Пирогова» Министерства здравоохранения Российской Федерации; e-mail: alimovaee@yandex.ru

\section{PEзЮMЕ}

Декслансопразол MB является R-энантиомером лансопразола и в настоящее время является единственным ингибитором протонного насоса с новой технологией доставки Dual Delayed Release. Фармакокинетика препарата показывает два пика высвобождения в двенадцатиперстной и тонкой кишках; антисекреторное действие показано в дозах 30 и 60 мг, удержание внутрижелудочного $\mathrm{pH}>4$ более 16,5 ч/сут независимо от времени приема. В клинических исследованиях показано, что декслансопразол МВ обладает высокой эффективностью в заживлении эрозивного эзофагита и контроле симптомов у пациентов с неэрозивной рефлюксной болезнью, поддерживает ремиссию при длительном применении. Эффективен в улучшении симптомов ночной изжоги, связанной с ГЭРБ, при нарушении сна. Декслансопразол МВ имеет аналогичный профиль безопасности и побочных эффектов с лансопразолом.

Ключевые слова: декслансопразол МВ, фармакокинетика, фармакодинамика, антисекреторная активность, ГЭРБ, эрозивный эзофагит, неэрозивная болезнь

Для цитирования: Леонова М.В., Алимова Э.Э. Декслансопразол МВ в лечении гастроэзофагеальной рефлюксной болезни. Медицинский совет. 2018; 21: 132-137. DOI: https://doi.org/10.21518/2079-701X-2018-21-132-137.

Конфликт интересов: авторы заявляют об отсутствии конфликта интересов.

\section{Dexansoprazole MV}

\section{IN THE TREATMENT OF GASTROESOPHAGEAL REFLUX DISEASE}

\author{
Marina V. LEONOVA, Elmira E. ALIMOVA \\ Federal State Budgetary Educational Institution of Higher Education "Pirogov Russian National Research Medical University" of the Ministry
} of Health of Russia, Moscow 117997, Moscow, 1 Ostrovityanova,

\section{Author credentials:}

Leonova Marina Vasilievna - Dr. of Sci. (Med.), Professor, Corr. Member of RANS, Clinical Pharmacologist, Member of the Interregional Public Organization, Association of Clinical Pharmacologists of Russia; tel.: +7 (915) 320-43-79; e-mail: anti23@mail.ru

Alimova Elmira Erfanovna - Cand.of Sci. (Med.), Associate Professor, Department of Clinical Pharmacology, Faculty of General Medicine, Federal State Budgetary Educational Institution of Higher Education «Pirogov Russian National Research Medical University» of the Ministry of Health of the Russian Federation; e-mail: alimovaee@yandex.ru

\section{ABSTRACT}

Dexlensoprazole MB is the R-enantiomer of lansoprazole and is currently the only proton pump inhibitor with the new Dual Delayed Release delivery technology. The pharmacokinetics of the drug shows two peaks of release in the 12-finger and small intestine; antisecretory effect is shown in doses of $30 \mathrm{mg}, 60 \mathrm{mg}$, and retention of intragastric $\mathrm{pH}>4$ more than 16.5 hours per day, regardless of the time of administration. Clinical studies have shown that dexlansoprazole MB is highly effective in the healing of erosive esophagitis and symptom control in patients with non-erosive reflux disease, maintains remission with prolonged use. Effective in improving the symptoms of night heartburn associated with GERD, with sleep disturbances. Dexlensoprazole MB has a similar safety profile and side effects with lansoprazole.

Keywords: Dexlensoprazole MR, pharmacokinetics, pharmacodynamics, antisecretory activity, GERD, erosive esophagitis, nonerosive disease

For citing: Leonova M.V., Alimova E.E. Dexansoprazole MV in the treatment of gastroesophageal reflux disease. Meditsinsky Sovet. 2018; 21: 132-137. DOI: https://doi.org/10.21518/2079-701X-2018-21-132-137. 
П оявление и внедрение ингибиторов протонной помпы (ИПП) в клиническую практику в начале 1990-х гг. коренным образом изменило возможность консервативного лечения кислот-зависимых заболеваний ЖКТ. Фармакологическое подавление продукции соляной кислоты препаратами ИПП показало высокую эффективность в лечении язвенной болезни и гастроэзофагеальной рефлюксной болезни (ГЭРБ) и минимизировало потребность в хирургическом лечении этих заболеваний. Важное практическое значение применение ИПП имеет также в профилактике и лечении НПВП-ассоциированной гастропатии, для профилактики стресс-язв в отделениях интенсивной терапии, в лечении кровотечений из верхних отделов ЖКТ.

Несмотря на то что ИПП в настоящее время превосходят по антисекреторному действию все другие классы препаратов, в первую очередь $\mathrm{H}_{2}$-блокаторы, продолжается разработка новых препаратов этого фармакологического класса с целью более длительного и эффективного контроля кислотопродукции.

Ряд проблем остается в фармакотерапии кислот-зависимых заболеваний ЖКТ. К ним относятся лечение пациентов с симптомами ГЭРБ, которые неадекватно отвечают на терапию ИПП.

Несмотря на успех ИПП в лечении пациентов с эрозивным эзофагитом, по-прежнему существуют серьезные проблемы в лечении пациентов с ГЭРБ. По данным Кокрейновского метаанализа установлено, что 19-44\% пациентов не отвечают адекватно на терапию ИПП, что проявляется рефлюксными симптомами изжоги $[1,2]$. Применение ИПП в реальной клинической практике неэффективно примерно у $30 \%$ пациентов с эрозивным эзофагитом, имеющих многочисленные и обширные эрозии или язвы, и обычно эти пациенты испытывают более частые проявления гастроэзофагеального рефлюкса, особенно в ночное время [3]. Доля пациентов с неэрозивной рефлюксной болезнью (НЭРБ), которые неадекватно реагируют на терапию ИПП, значительно выше, чем пациентов С эрозивным эзофагитом, и может составлять до $60 \%$ всех больных ГЭРБ.

Для успешного лечения эзофагита или ГЭРБ были описаны три главных фактора: степень подавления кислотности, продолжительность подавления в течение 24-часового периода и длительность лечения в неделях. Кроме того, эти три фактора отражают общее время, в течение которого остается рН пищевода > 4 в течение периода антисекреторной терапии. Для нормализации внутриэзофагеальной кислоты требуется длительное поддержание $\mathrm{pH}$ пищевода > 4 и уменьшение периода $\mathrm{pH}<$ 4 не более 4\% за 24 ч (правило Белла) [4]. Лечение препаратами ИПП, основанное на вышеуказанных принципах, приводило к высокой частоте заживления эрозивного эзофагита вплоть до 90\% через 8 недель. Однако у пациентов с более тяжелыми проявлениями эзофагита частота заживления более низкая (62-84\%) [5].

Более того, в исследованиях было показано, что значительная часть пациентов с ГЭРБ не полностью удовлетворена антисекреторной терапией. По данным опросного исследования 1013 пациентов с ГЭРБ, применяющих ИПП, более $35 \%$ с приемом один раз в день и 54\% с приемом дважды в день указали, что терапия не полностью устраняет симптомы [6]. Причем, если частота заживления эрозивного эзофагита достигается у большинства пациентов после 8 недель лечения ИПП, меньший ответ наблюдается по устранению симптомов изжоги (>60\%) [2].

Проблемой эффективного лечения остается НЭРБ. В систематическом обзоре Dean B.B. общая частота не ответивших на терапию ИПП составила $63 \%$ среди пациентов с НЭРБ по сравнению с 44\% для пациентов с эрозивным эзофагитом после 4 недель терапии ИПП ( $<<0,0001)$ [7]. Результаты крупного метаанализа Zhang J.X. также подтвердили невысокий уровень эффективности ИПП у пациентов с НЭРБ - лишь в 51,5\% [8].

Показано, что прием стандартной дозы ИПП один раз в день ингибирует примерно 75\% активных протонных насосов, для достижения максимального подавления кислоты может потребоваться до 3 дней [9]. В сравнительном исследовании стандартных доз ИПП было установлено, что средняя продолжительность рН желудка > 4 была сопоставимой для омепразола 20 мг (11,8 4), лансопразола 30 мг (11,5 4), пантопразола 40 мг (10,1 ч) и рабепразола 20 мг (12,1 ч), значительно дольше для эзомепразола 40 мг (14,0 ч) [10]. В этой связи рекомендуется удвоение разовой дозы ИПП или двукратный прием препаратов для увеличения эффективности при ГЭРБ [11, 12].

Зачастую именно ночные симптомы ГЭРБ плохо купируются при приеме ИПП, что приводит к нарушению сна и существенно ухудшает качество жизни пациентов [13], и около 70\% пациентов с ГЭРБ показывают явление ночного кислотного прорыва при приеме ИПП 2 раза в день [14].

\section{ФАРМАКОЛОГИЯ, ФАРМАКОДИНАМИКА И ФАРМАКОКИНЕТИКА ДЕКСЛАНСОПРАЗОЛА МВ}

Все доступные в настоящее время ИПП: эзомепразол, омепразол, лансопразол, пантопразол и рабепразол являются замещенными бензимидазолами и пролекарствами. Они представляют собой кислотолабильные, слабые основания, которые требуют защиты от активации в кислой среде желудка до того, как они достигнут своего основного места абсорбции в проксимальном тонком кишечнике. Практически все лекарственные формы ИПП являются препаратами с отсроченным высвобождением для защиты от деградации в желудке, что достигается с помощью кишечнорастворимой оболочки [15].

Декслансопразол МВ представляет новую лекарственную форму ИПП с модифицированным высвобождением и пролонгированным действием [16]. Этот эффект достигается через два механизма: использование активного вещества R-энантиомера лансопразола и технологию системы доставки Dual Delayed Release.

Лансопразол имеет два энантиомера (S- и R-), которые являются эквипотенциальными ингибиторами протонного насоса. R-энантиомер, или декслансопразол, представляет более $80 \%$ циркулирующего лекарственного средства после перорального приема лансопразола и отлича- 
Рисунок 1. Средние профили концентрации в плазме для декслансопразола МВ и лансопразола у здоровых добровольцев (5-й день) в исследованиях фармакокинетики [20] - Figure 1. Mean plasma concentration profiles for dexlansoprazole MV and lansoprazole in healthy subjects (Day 5) in the pharmacokinetics studies [20]

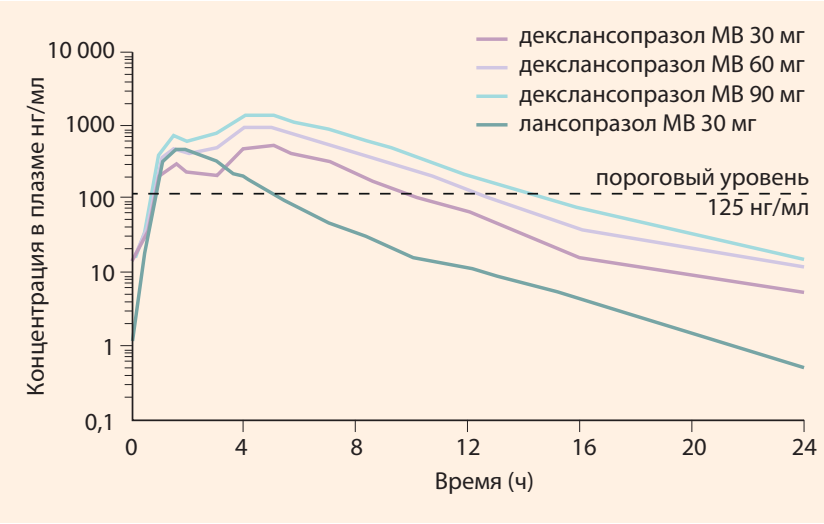

ется более медленным клиренсом и большим значением AUC, чем S-энантиомер [17].

Важным достижением была разработка декслансопразола МВ с использованием новой формулы декслансопразола в форме с модифицированным высвобождением, в которой используется технология Dual Delayed Release, предназначенная для пролонгирования профиля концентрации декслансопразола и обеспечения длительной продолжительности подавления кислоты [16, 18]. В этой технологии двойного замедленного высвобождения используются два типа гранул лекарственного вещества с кишечнорастворимыми оболочками, имеющими различные $\mathrm{pH}$-зависимые профили растворения. После приема внутрь наружная желатиновая капсула, содержащая гранулы, растворяется в желудке, и первый тип гранул ( 25\% дозы) высвобождается в двенадцатиперстную кишку с рН 5,5. Это приводит к раннему повышению концентрации в плазме через 1-2 ч (первый пик), аналогичному другим ИПП. Второй тип гранул ( 75\% дозы) предназначен для высвобождения в дистальном тонком кишечнике с рН 6,75, что приводит ко второму пику концентрации через 4-5 ч после приема внутрь, что подтверждается исследованиями фармакокинетики [19]. Такая система доставки обеспечивает длительную продолжительность подавления кислоты и помогает ограничить необходимость дозирования более одного раза в день [16].

По результатам исследований фармакокинетики для поддержания фармакологически активных концентраций декслансопразола в плазме требуется более высокая суточная доза декслансопразола МВ по сравнению с лансопразолом с обычным высвобождением [19]. Используя фармакокинетические/фармакодинамические модели, было установлено, что уровень концентрации и дозы декслансопразола для достижения процента времени, в течение которого поддерживается 24-часовой внутрижелудочный $\mathrm{pH}>$ 4, составляет 125 нг/мл. Наряду с этим, была определена пороговая концентрация, обеспечивающая средний уровень рH > 4,49 (рис. 1) [20]. В фармако- кинетическом исследовании показано, что в диапазоне доз декслансопразола МВ от 30 до 90 мг препарат поддерживает концентрацию лекарственного средства в плазме выше, чем пороговый уровень 125 нг/мл, а также в 2-3 раза дольше, чем лансопразол в дозе 30 мг.

Основываясь на результатах исследований фармакодинамики, прогнозируемые средние значения 24-часового внутрижелудочного рН составляли 4,06 и 4,35, а значение pH > 4 в течение 24 ч составляло 59,2 и 66,7\% для декслансопразола MR в дозах 30 и 90 мг соответственно [19].

В перекрестном исследовании у 48 здоровых добровольцев с ГЭРБ изучалась фармакокинетика и фармакодинамика декслансопразола МВ 60 мг в зависимости от режима приема: за 30 мин перед завтраком, ланчем, обедом или ужином на протяжении 5 дней [21]. Всасывание препарата нарушалось во всех случаях в сравнении с приемом перед завтраком, вызывая замедление времени $\mathrm{T}_{\max }$ (латентный период, или $\mathrm{t}_{\text {lag }}$ ) на 2-3 ч при приеме перед ланчем, обедом или ужином ( $<<0,05)$ (рис. 2). При этом два пика концентрации декслансопразола в соответствии с фазами высвобождения препарата из лекарственной формы определялись только при приеме перед завтраком или ланчем. Однако, несмотря на различия по показателям абсорбции, экспозиция препарата по показателям $C_{\max }$ и AUC (площадь под кривой «концентрация время») достоверно не различалась, указывая на отсутствие влияния на биодоступность декслансопразола при разных режимах приема. Мониторинг 24-часового внутрижелудочного рН показал, что после приема декслансопразола МВ перед завтраком уже через 1 ч обеспечивается уровень $\mathrm{pH}>4$ и удерживается на протяжении большей части 24-часового интервала, за исключением ночных часов. При приеме препарата перед обедом или ужином средние профили рН поддерживались выше значения 4 в течение большей части 24-часового интервала, но прием перед ужином давал отсрочку наступления эффекта более 6 ч. Средний процент внутрижелудочного $\mathrm{pH}>4$ в течение 24-часового интервала на 5-й день составлял 71, 74, 70\% при приеме перед завтраком, ланчем, обедом и 64\% при приеме перед ужином, что было статистически меньшим ( $p=0,016)$. Средний внутрижелудочный $\mathrm{pH}$ после приема декслансопразола МВ был наибольшим перед ланчем -

- Рисунок 2. Сравнение фармакокинетических кривых после приема декслансопразола МВ 60 мг перед приемом пищи [21]

- Figure 2. Comparison of pharmacokinetic curves after administration of dexlansoprazole MV $60 \mathrm{mg}$ before meals [21]

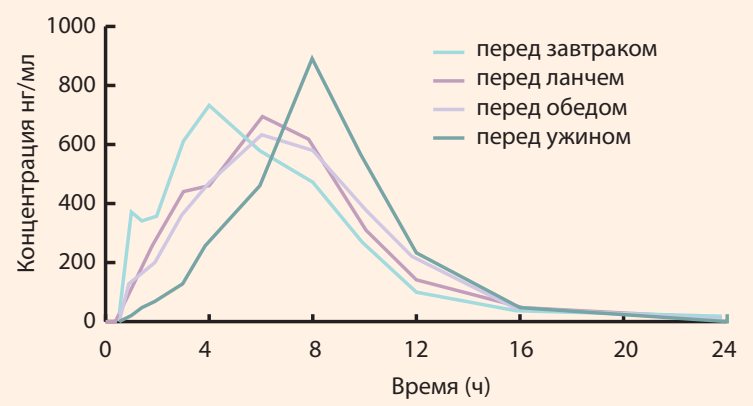


4,83 против 4,63 перед завтраком, 4,63 перед обедом и 4,60 перед ужином ( $p<0,05)$ [21]. Таким образом, выявленное нарушение всасывания декслансопразола МВ при приеме перед дневным или вечерним приемом пищи не влияет значимо на антисекреторное действие и не является клинически значимым, обеспечивая удержание внутрижелудочного рH>4 более 16,5 ч/сут.

\section{КЛИНИЧЕСКАЯ ЭФФЕКТИВНОСТЬ ДЕКСЛАНСОПРАЗОЛА МВ В ЛЕЧЕНИИ ГЭРБ}

Проведено прямое сравнение клинической эффективности декслансопразола MB и лансопразола в двух двойных слепых исследованиях у 4092 пациентов с ГЭРБ [22]. Частота заживления эрозивного эзофагита через 8 недель лечения на фоне применения декслансопразола МВ 60 мг 1 раз в сутки была сопоставимой с обычной формой лансопразола 30 мг 1 раз в сутки и составила 92-95\%. При этом интегрированный анализ результатов лечения в подгруппе пациентов с умеренным и тяжелым течением ГЭРБ показал преимущество декслансопразола МВ перед лансопразолом: дополнительно 25-30\% пациентов, которые не отвечают на лечение в течение 8 недель, могут достичь эффективности на фоне лечения декслансопразолом МВ [22].

В ряде исследований изучалась эффективность декслансопразола МВ в лечении ГЭРБ. Так, в двойном слепом исследовании у 947 пациентов с ГЕРБ оценивали частоту наступления 24-часовых дней без изжоги (первичная точка) и ночей без изжоги (вторичная точка) через 4 недели приема декслансопразола МВ в дозах 30 и 60 мг однократно в сутки [23]. Декслансопразол MR обеспечивал значительно больший средний процент из 24-часовых дней без изжоги (54,9 и 50,0\% для 30 мг и 60 мг против 17,5\% для плацебо, p<0,00001) и ночей без изжоги (80,8 и 76,9\% против 51,7\% для плацебо, p<0,00001) (рис. 3). Препарат также уменьшал тяжесть симптомов [23].

Среди пациентов с симптомами ГЭРБ до 89\% сообщают о ночных симптомах изжоги, что приводит к ухудшению качества сна и качества жизни, связанного со здоровьем, а в дневное время - к снижению активности и производительности [24, 25]. Декслансопразол МВ, благодаря пролонгированию антисекреторного эффекта,

- Рисунок 3. Снижение частоты 24-часовых дней и ночей без изжоги при приеме декслансопразола MB [23]

- Figure 3. Dexlansoprazole MW reduced the frequency of 24-hour heartburn-free days and heartburn-free nights [23]
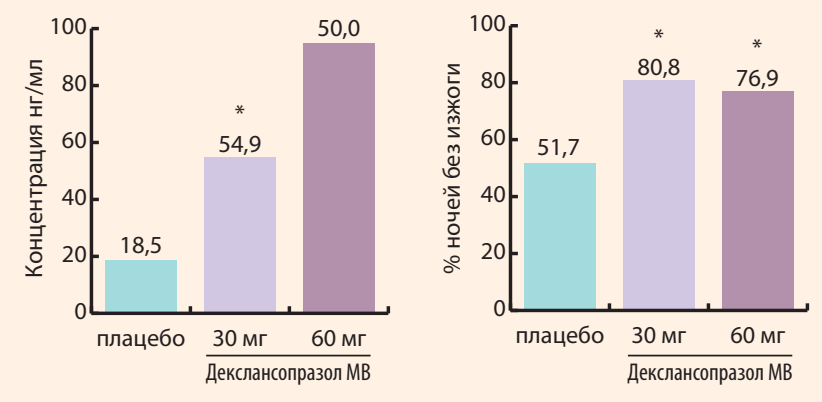

Рисунок 4. Процент ночей с нарушениями сна, связанными с ГЭРБ, по оценке пациентами в ежедневном дневнике на фоне лечения декслансопразолом MB [26]

- Figure 4. Percentage of nights with GERD-related sleep disturbances as assessed by patient daily diary over treatment with dexlansoprazole MV [26]

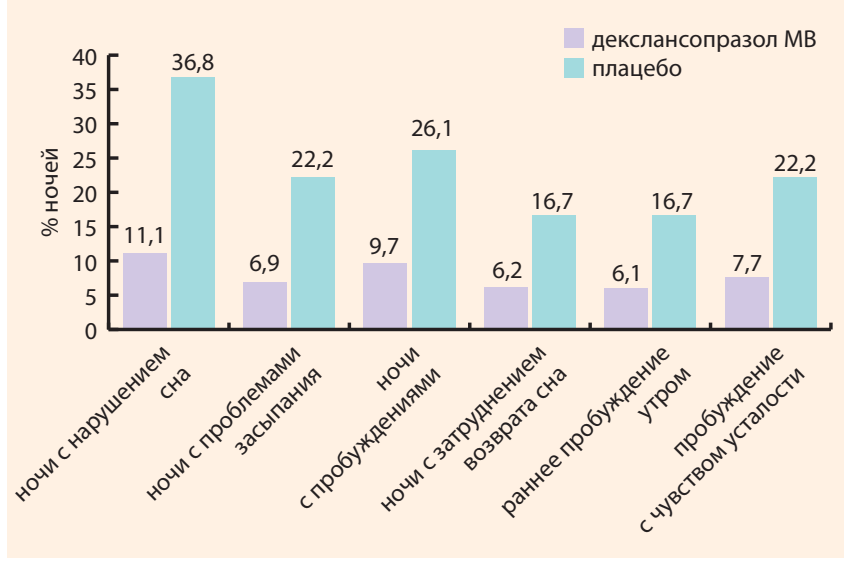

позволяет облегчить ночную симптоматику и связанные с ночной изжогой нарушения сна у пациентов с ГЭРБ. В исследовании у 305 больных, имеющих умеренные и тяжелые симптомы ночной изжоги и расстройства сна, на фоне приема препарата в дозе 30 мг в течение 4 недель на 73,1\% уменьшалась частота ночной изжоги против $35,7 \%$ на фоне плацебо (p<0,001) и на 47,5\% снижалась частота ГЭРБ-ассоциированных нарушений сна против $19,6 \%$ на плацебо ( $p<0,001)$. Почти в 3 раза снижалась частота ночей с нарушением сна, с нарушением засыпания, с ночным или ранним пробуждением (рис. 4) [26].

Известно, что многие пациенты с ГЭРБ для лучшего контроля симптомов изжоги применяют препараты ИПП два раза в сутки. В этой связи было проведено исследование по возможности step-down-терапии ИПП с применением декслансопразола МВ для однократного приема. Было включено 178 пациентов с ГЭРБ, которые применяли любой препарат ИПП два раза в сутки и имели хороший контроль симптомов изжоги (не более одного симптома в неделю в течение последних 4 недель лечения) [27]. Пациенты слепым способом были переведены на декслансопразол МВ 30 мг и плацебо для лечения в течение 6 недель. Контроль симптомов изжоги пациенты фиксировали два раза в день в электронных дневниках. Первичной конечной точкой эффективности была доля пациентов, изжога которых оставалась хорошо контролируемой после снижения кратности ИПП при переводе на декслансопразол МВ. Оценивалась также тяжесть симптомов и качество жизни. После перевода пациентов на однократный прием декслансопразола МВ 30 мг изжога оставалась хорошо контролируемой у 88\% пациентов; статистически значимо уменьшалась тяжесть симптомов вздутия и изжоги и улучшались показатели качества жизни [27]. Таким образом, лекарственная форма декслансопразола МВ с двойным высвобождением приводит к увеличению продолжительности подавления кислотопродукции и может способствовать успешной step-down-терапии ИПП. Эти результаты 
Таблица. Сравнение побочных эффектов при приеме декслансопразола MB, лансопразола и плацебо у пациентов с ГЭРБ [16]

Table. Comparison of side effects following administration of dexansoprazole MV, lansoprazole and placebo in patients with GERD [16]

\begin{tabular}{l|c|c|c|c}
\hline Побочные эффекты (\%) & Декслансопразол МВ 30 мг & Декслансопразол МВ 60 мг & Лансопразол 30 мг & Плацебо \\
\hline Диарея & 5,1 & 4,8 & 3,2 & 2,9 \\
\hline Боль в животе & 3,5 & 4,0 & 2,6 & 2,5 \\
\hline Тошнота & 3,3 & 2,8 & 1,8 & 0,8 \\
\hline Инфекция ВДП & 2,9 & 1,7 & 0,8 & 0,8 \\
\hline Рвота & 2,2 & 1,4 & 1,1 & 0,6 \\
\hline Метеоризм & 2,6 & 1,4 & 1,2 & \\
\hline
\end{tabular}

Примечание. ВДП - верхние дыхательные пути.

могут быть широко применены к реальной клинической практике для длительного контроля симптомов изжоги.

Проблемой лечения ГЭРБ является не только достижение заживления эрозивного эзофагита, но и сохранение ремиссии и длительное поддержание контроля симптомов. Вместе с тем после заживления продолжение приема препаратов ИПП все равно сопровождается обострением от 15 до 41\% в течение 6 месяцев [28]. Изучалась эффективность лечения декслансопразолом МВ в поддержании достигнутого заживления эрозивного эзофагита и облегчении изжоги.

Проведено крупное двойное слепое исследование у 445 пациентов с залеченным эрозивным эзофагитом, которые продолжали принимать декслансопразол МВ в дозах 30 мг или 60 мг плацебо один раз в день в течение 6 месяцев [29]. Критериями эффективности было поддержание эндоскопического заживления (первичная точка) и продолжение облегчения симптомов на основе ежедневных дневников (вторичная точка), в которых регистрировали выраженность и тяжесть дневных и ночных симптомов по 5-балльной шкале. По эндоскопической оценке у $66,4 \%$ в группе декслансопразола МВ сохранялась ремиссия против 14,3\% в группе плацебо ( $p<0,00001)$ (рис. 5). Среди пациентов, у которых наблюдался рецидив эрозив-

Рисунок 5. Совокупная частота поддержания ремиссии эрозивного эзофагита у пациентов на фоне лечения декслансопразолом МВ в течение 6 месяцев [29]

- Figure 5. The cumulative frequency of maintaining remission of erosive esophagitis in patients against the background of treatment with dexlansoprazole MB for 6 months [29]

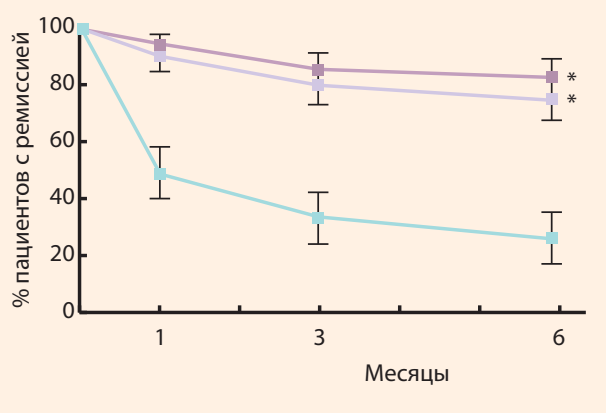

плацебо декслансопразол $\mathrm{MB} 30 \mathrm{Mr}$ декслансопразол MB 60 Mг ного эзофагита, среднее время до рецидива было значительно большим для группы декслансопразола МВ против группы плацебо (42-й и 61-й день, $p=0,0015)$. Процент 24-часовых дней без изжоги, по данным дневников пациентов, был значительно выше в каждой группе лечения декслансопразолом МВ, чем в группе плацебо (91 и 96\% для доз 30 и 60 мг против 29\% соответственно, $p<0,0025)$. Процент ночей без изжоги был также значительно выше при лечении декслансопразолом МВ (96 и 99\% для доз 30 и 60 мг против 72\% соответственно, р<0,0025) [29].

\section{ПЕРЕНОСИМОСТЬ И БЕЗОПАСНОСТЬ ДЕКСЛАНСОПРАЗОЛА МВ}

По данным 6 контролируемых исследований и 12-месячного исследования ( $n=4270)$, декслансопразол МВ в диапазоне доз 30-90 мг хорошо переносится и имеет профиль безопасности, подобный лансопразолу [30]. Отмечено меньшее количество побочных эффектов на 100 пациентов/ месяцев в группе декслансопразола МВ по сравнению с группами лансопразола и плацебо (15,64-18,75 против 21,06 и 24,49 соответственно). Наиболее частым побочным эффектом была диарея, боли в животе, тошнота, инфекция верхних дыхательных путей, рвота и метеоризм (табл.) [16].

Повышение концентрации гастрина в плазме при применении ИПП относится к хорошо известному классэффекту и обусловлено компенсаторным увеличением продукции гормона в ответ на подавление кислотообразования. Это один из вопросов безопасности длительного применения ИПП. В исследовании у здоровых добровольцев средний уровень $\mathrm{AUC}_{24}$ гастрина увеличивался примерно в 3,5 раза по сравнению с исходными значениями после 5 дней приема декслансопразола МВ в дозах 90 или 120 мг; эта величина была сопоставимой с таковой после приема лансопразола в дозе 30 мг [31]. При проведении биопсии желудка у пациентов и излеченным эрозивным эзофагитом не было выявлено различий для любых доз декслансопразола МВ. В долгосрочном исследовании безопасности наиболее часто встречался хронический гастрит у 31\% пациентов на фоне приема 60 мг декслансопразола MB; не было сообщений о гиперплазии ECL в исследованиях продолжительностью от 6 месяцев до 1 года [30]. 


\section{ЗАКЛЮЧЕНИЕ}

Применение препаратов ИПП является препаратами выбора в лечении ГЭРБ [32]. Однако существуют проблемы недостаточной эффективности лечения и неудовлетворенности лечением пациентов при приеме препаратов ИПП. Для решения проблем был разработан новый препарат с модифицированным высвобождением Dual Delayed Release - декслансопразол МВ.

Декслансопразол МВ, благодаря особенностям активного вещества и технологии доставки, отличается пролонгированным этапом всасывания в ЖКТ, что обе- спечивает более длительный антисекреторный эффект и оптимальное удержание внутрижелудочного $\mathrm{pH}>4$ более 16,5 ч/сут при любом режиме приема. Эффективными дозами препарата являются 30 и 60 мг, которые имеют терапевтический уровень концентрации. Такие характеристики лекарственной формы декслансопразола МВ имеют преимущества перед обычными формами препаратов ИПП в лечении пациентов с ГЭРБ как при курсовом применении для заживления эрозивного эзофагита и лечении изжоги у пациентов с неэрозивным эзофагитом, так и при длительном контроле симптомов заболевания.

\section{ЛИTEPATYPA/REFERENCES}

1. Moayyedi P., Santana J., Khan M., Preston C., Donnellan C. WITHDRAWN: Medical treatments in the short term management of reflux oesophagitis. Cochrane Database Syst Rev. 2011; 2: CD003244.

2. Katz P.O., Scheiman J.M., Barkun A.N. Review article: Acid-related disease - What are the unmet clinical needs? Aliment Pharmacol Ther. 2006; 23(Suppl 2): 9-22.

3. Ruigómez A., Johansson S., Wernersson B., Fernández C.O., García R.L.A.. Gastroesophageal reflux disease in primary care: using changes in proton pump inhibitor therapy as an indicator of partial response. Scand J Gastroenterol. 2012; 47(7): 751-61.

4. Bell N.J., Burget D., Howden C.W., Wilkinson J., Hunt R.H. Appropriate acid suppression for the management of gastro-oesophageal reflux disease. Digestion. 1992, 51(Suppl. 1): 59-67.

5. Hunt R.H. Review article: the unmet needs in delayed-release proton-pump inhibitor therapy in 2005. Aliment Pharmacol Ther. 2005, 22(Suppl. 3): 10-19.

6. Chey W.D., Mody R.R., Izat E. Patient and physician satisfaction with proton pump inhibitors (PPIs): are there opportunities for improvement? Dig Dis Sci. 2010; 55(12): 3415-22.

7. Dean B.B., Gano A.D., Knight K., Ofman J.J., Fass R. Effectiveness of proton pump inhibitors in nonerosive reflux disease. Clin Gastroenterol Hepatol. 2004; 2: 656-64.

8. Zhang J.X., Ji M.Y., Song J., Lei H.B., Qiu S., Wang J. et al. Proton pump inhibitor for non-erosive reflux disease: a meta-analysis. World J Gastroenterol. 2013; 19(45): 8408-19.

9. Sachs G., Shin J.M., Howden C.W. Review article: the clinical pharmacology of proton pump inhibitors. Aliment Pharmacol Ther. 2006; 23(Suppl 2): 2-8.

10. Miner P.J., Katz P.O., Chen Y., Sostek M. Gastric acid control with esomeprazole, lansoprazole, omeprazole, pantoprazole, and rabeprazole: a five-way crossover study. Am J Gastroenterol. 2003; 98: 2616-20.

11. Fass R., Murthy U., Hayden C.W., Malagon I.B., Pulliam G., Wendel C., Kovacs T.O. Omeprazole $40 \mathrm{mg}$ once a day is equally effective as lansoprazole $30 \mathrm{mg}$ twice a day in symptom control of patients with gastro-oesophageal reflux disease (GERD) who are resistant to conventionaldose lansoprazole therapy-a prospective, randomized, multi-centre study. Aliment Pharmacol Ther. 2000; 14(12): 1595-603.

12. Zhang H., Yang Z., Ni Z., Shi Y. A meta-analysis and systematic review of the efficacy of twice daily PPIs versus once daily for treatment of dastroesophageal reflux disease. Gastroenterol Res Pract. 2013; Article ID 9865963.
13. Fujiwara Y., Arakawa T., Fass R. Gastroesophageal reflux disease and sleep disturbances. J Gastroenterol. 2012; 47(7): 760-9.

14. Nzeako U.C., Murray J.A. An evaluation of the clinical implications of acid breakthrough in patients on proton pump inhibitor therapy. Aliment Pharmacol Ther. 2002; 16: 1309-16.

15. Белоусов Ю.Б., Леонова М.В. Лекарственные формы с модифицированным высвобождением и системы доставки лекарств: особенности фармакокинетики и клиническая эффективность. М.: «Литтерра», 2011: 440-444. [Belousov Yu.B., Leonova M.V. Modified release dosage forms and drug delivery systems: pharmacokinetic features and clinical efficacy. M.: Litterra, 2011: 440-444] (In Russ).

16. Frye J.W., Peura D.A. Managing gastroesophageal reflux disease - comparative efficacy and outcomes of dexlansoprazole MR. Ther Clin Risk Manag. 2015; 11: 1649-56.

17. Katsuki H., Yagi H., Arimori K., Nakamura C., Nakano M., Katafuchi S. et al. Determination of $\mathrm{R}(+)$ - and $\mathrm{S}(-)$-lansoprazole using chiral stationary-phase liquid chromatography and their enantioselective pharmacokinetics in humans. Pharm Res. 1996, 13: 611-5.

18. Metz D.C., Vakily M., Dixit T., Mulford D. Dual delayed release formulation of dexlansoprazole MR, a novel approach to overcome the limitations of conventional single release proton pump inhibitor therapy. Aliment Pharmacol Ther. 2009; 29: 928-37.

19. Vakily M., Zhang W., Wu J., Atkinson S., Mulford D. Pharmacokinetics and pharmacodynamics of a known active PPI with a novel Dual Delayed Release technology, dexlansoprazole MR: a combined analysis of randomized controlled clinical trials. Curr Med Res Opin. 2009; 25: 627-38.

20. Wu J., Vakily M., Witt G., Mulford D. TAK-390MR vs. lansoprazole (LAN) for maintenance of drug concentration above a threshold which corresponds to higher\%-time $\mathrm{pH}>4$. Am J Gastroenterol. 2007; 102(suppl 2): S124 (abstract).

21. Lee R.D., Mulford D., Wu J., Atkinson S.N. The effect of time-of-day dosing on the pharmacokinetics and pharmacodynamics of dexlansoprazole MR: evidence for dosing flexibility with a dual delayed release proton pump inhibitor. Aliment Pharmacol Ther. 2010; 31: 1001-11.

22. Sharma P., Shaheen N.J., Perez M.C., Pilmer B.L., Lee M., Atkinson S.N., Peura D. Clinical trials: healing of erosive oesophagitis with dexlansoprazole MR, a proton pump inhibitor with a novel delayed-release formulation - results from two randomized controlled studies. Aliment Pharmacol Ther. 2009; 29: 731-41.

23. Fass R., Chey W.D., Zakko S.F, Andhivarothai N., Palmer R.N., Perez M.C., Atkinson S.N. Clinical trial: the effect of the proton pump inhibitor dexlansoprazole MR on daytime and nighttime heartburn in patients with non-erosive reflux disease. Aliment Pharmacol Ther. 2009; 29: 1261-72.

24. Farup C., Kleinman L., Sloan S., Ganoczy D., Chee E., Lee C., Revicki D. The impact of nocturnal symptoms associated with gastroesophageal reflux disease on health-related quality of life. Arch Intern Med. 2001; 161: 45-52

25. Mody R., Bolge S.C., Kannan H., Fass R. Effects of gastroesophageal reflux disease on sleep and outcomes. Clin Gastroenterol Hepatol. 2009; 7: $953-9$.

26. Fass R., Johnson D.A., Orr W.C., Han C., Mody R., Stern K.N. et al. The effect of dexlansoprazole MR on nocturnal heartburn and GERD-related sleep disturbances in patients with symptomatic GERD. Am J Gastroenterol. 2011; 106: 421-31.

27. Fass R., Inadomi J., Han G., Mody R., O’Neil J., Perez M.C. Maintenance of heartburn relief after stepdown from twice-daily proton pump inhibitor to once-daily dexlansoprazole modified release. Clin Gastroenterol Hepatol. 2012; 10: 247-63.

28. Dickman R., Maradey-Romero C., Gingold-Belfer R., Fass R.. Unmet needs in the treatment of gastroesophageal reflux disease. J Neurogastroenterol Motil. 2015; 21: 309-19.

29. Metz D.C., Howden C.W., Perez M.C., Larsen L., O'Neil J., Atkinson S.N. Clinical trial: dexlansoprazole MR, a proton pump inhibitor with dual delayed-release technology, effectively controls symptoms and prevents relapse in patients with healed erosive oesophagitis. Aliment Pharmacol Ther. 2009; 29: 742-54.

30. Peura D.A., Metz D.C., Dabholkar A.H., Paris M.M., Yu P., Atkinson S.N. Safety profile of dexlansoprazole MR, a proton pump inhibitor with a novel dual delayed release formulation: global clinical trial experience. Aliment Pharmacol Ther. 2009; 30: 1010-21.

31. Zhang W., Wu J., Atkinson S.N. Effects of dexlansoprazole MR, a novel dual delayed release formulation of a proton pump inhibitor, on plasma gastrin levels in healthy subjects. J Clin Pharmacol. 2009; 49(4): 444-454.

32. Ивашкин В.Т., Маев И.В., Трухманов А.С., Баранская Е.К., Дронова О.Б., Зайратьянц О.В. и др. Клинические рекомендации Российской гастроэнтерологической ассоциации по диагностике и лечению гастроэзофагеальной рефлюксной болезни. Рос. журн. гастроэнтерол. гепатол. колопроктол. 2017; 27(4): 75-95. [Ivashkin V.T., Maev I.V., Trukhmanov A.S., Baranskaya E.K., Dronova O.B., Zayratyants O.V., et al. Clinical guidelines of the Russian Gastroenterological Association for the diagnosis and treatment of gastroesophageal reflux disease. Ros. Zhurn. Gastroenterol. Gepatol. Koloproktol 2017; 27 (4): 75-95] (In Russ). 